\title{
Residência em Enfermagem Obstétrica na formação do líder: um relato de experiência
}

Residency in Obstetric Nursing in the training of the leader: an experience report

Residencia en Enfermería Obstétrica en la formación del líder: un relato de experiencia

Recebido: 25/05/2021 | Revisado: 31/05/2021 | Aceito: 04/09/2021 | Publicado: 06/09/2021

Maria Odete Queiroz Lima Tavares

ORCID: https://orcid.org/0000-0003-4399-1409

Universidade Estadual de Ciências da Saúde de Alagoas, Brasil E-mail: odetequeiroz34@gmail.com

Sandra Taveiros de Araújo

ORCID: https://orcid.org/0000-0003-1286-1759

Universidade Estadual de Ciências da Saúde de Alagoas, Brasil

E-mail: taveirossandra@gmail.com

Maria Elisângela Torres de Lima Sanches

ORCID: https://orcid.org/0000-0001-8987-3825

Universidade Federal de Alagoas, Brasil E-mail: eli_sanches23@hotmail.com

Ericássia Vanessa da Silva Albuquerque

ORCID: https://orcid.org/0000-0003-4786-3156

Universidade Estadual de Ciências da Saúde de Alagoas, Brasil

E-mail: ericassia_albuquerque@ outlook.com

Jamile Vieira dos Santos

ORCID: https://orcid.org/0000-0001-5746-4322

Universidade Estadual de Ciências da Saúde de Alagoas, Brasil

E-mail: mile.enfermagem2012@gmail.com

\begin{abstract}
Resumo
Objetivo: Conhecer as vivências de uma enfermeira obstétrica egressa pelo programa de residência enquanto líder de um Centro de Parto Normal interligando os conhecimentos e habilidades adquiridas durante a residência com sua implementação na liderança. Método: Trata-se de um estudo de natureza qualitativa e descritiva, do tipo relato de experiência que ocorreu durante a atuação como enfermeira obstetra entre a residência e em uma Maternidade de Risco Habitual, situada na região Nordeste do Brasil. Resultados e Discussão: As experiências vivenciadas durante o período da residência auxiliou a compreender e sanar as lacunas de formação acadêmica quanto a assistência a mulher, mas também abrangeram o gerenciamento da equipe, tomada de decisões, solução de conflitos, dentre outros, proveniente da formação de uma líder. Considerações finais: A formação como enfermeira obstétrica por meio da modalidade de residência cumpriu seus objetivos, uma vez que possibilitou a aquisição de experiência na assistência obstétrica, permitindo a melhoria do desempenho da egressa na sua assistência como também forneceu o suporte ao exercício da gestão em enfermagem, promovendo a articulação do ensino, pesquisa e assistência.
\end{abstract}

Palavras-chave: Enfermeiras obstétricas; Liderança; Conhecimento; Educação superior; Ensino.

\begin{abstract}
Objective: To understand the routine of an obstetric nurse, egressing from the residency program to the position of leadership in a normal birth center, linking the knowledge and skills acquired during the residency with their implementation within the leadership. Method: Presented as a qualitative and descriptive study, a report of the real experience of performing the duties of an obstetric nurse between the residency and regular work in a normal risk maternity hospital, located in the northeast region of Brazil. Results and discussion: Undergoing the experiences of the residency period helped to comprehend and restore the gaps in the academic education regarding woman's assistance, but also encompassing team management, decision making, conflict mediation, among others, originating from a leadership education. Final considerations: The obstetric nurse education via the residency program accomplished its goals, as it allowed the acquiring of experience in the field obstetric assistance, permitting the improvement of performance of the egressed nurse in her assistance, as well as providing support in the exercise of nursing management, promoting teaching articulation, research and assistance.
\end{abstract}

Keywords: Obstetric nurses; Leadership; Knowledge; University education; Teaching. 


\begin{abstract}
Resumen
Objetivo: Conocer las experiencias de una enfermera obstétrica egresada del programa de residencia como líder de un Centro de Parto Normal, vinculando los conocimientos y habilidades adquiridos durante la residencia con su implementación en liderazgo. Método: Se trata de un estudio cualitativo y descriptivo, el tipo de relato de experiencia que ocurrió mientras trabajaba como enfermera obstétrica entre la residencia y en una Maternidad de Riesgo Habitual, ubicada en la región Nordeste de Brasil. Resultados y Discusión: Las experiencias vividas durante el período de residencia ayudaron a comprender y llenar los vacíos en la formación académica en materia de atención a la mujer, pero también abarcaron la gestión de equipos, toma de decisiones, resolución de conflictos, entre otros, derivados de la formación de un líder. Consideraciones finales: La formación como enfermera obstétrica a través de la modalidad de residencia cumplió con sus objetivos, ya que posibilitó la adquisición de experiencia en atención obstétrica, permitiendo la mejora del desempeño de la egresada en su cuidado, además de brindar apoyo al ejercicio de la gestión. en enfermería, promoviendo la articulación de la docencia, la investigación y la asistencia.
\end{abstract}

Palabras clave: Enfermeras obstétricas; Liderazgo; Conocimiento; Educación universitária; Ensenãnza.

\title{
1. Introdução
}

O cenário da assistência à mulher no parto tem sido influenciado por mudanças que ocorrem no Brasil, especialmente a mobilização, tanto das parturientes quanto das instituições de saúde e profissionais, em busca de uma assistência menos intervencionista e de um nascimento humanizado (Ferreira Jr et al., 2021).

O cuidado voltado para as necessidades da parturiente é potencializado a partir da conscientização das mulheres na luta pelos seus direitos e na reivindicação de melhorias das condições de vida. Nesse cenário, a Organização Mundial da Saúde (OMS) e o Fundo de População das Nações Unidas recomendam a liderança e o envolvimento de uma parteira ou enfermeira com habilidades de obstetrícia no pré-natal, assim como para o manejo do parto vaginal (Ferreira Jr et al, 2021).

À vista disso, para a efetivação desse novo modelo de atenção obstétrica e com a finalidade de provocar mudanças no modelo biomético intervencionista de assistência obstétrica, ainda presente no país, a gestão pública passa a estimular a inserção da enfermeira obstetra nas maternidades públicas.

A reorganização contínua do sistema de saúde para gerar respostas às demandas sociais exige adaptação da enfermagem para as novas realidades e pode ser vista como evolução profissional importante e necessária.

Nesse âmbito, os profissionais de saúde devem ter uma formação com efetividade e qualidade, seguindo os princípios e diretrizes do Sistema Único de Saúde (SUS), oportunizando uma atuação baseada nas necessidades sociais e de saúde e que esteja em conformidade com as Diretrizes Curriculares Nacionais (Antunes et al., 2017).

Concomitantemente, os cursos de graduação no país têm investido em profissionais que proponham mudanças dos modelos de atenção em saúde, através de inovações no processo de ensino-aprendizagem e no desenvolvimento de uma formação crítico-reflexiva de futuros trabalhadores (Oliveira et al., 2017).

Ressalta-se a relevância de uma gerência coerente com as competências, conhecimentos e habilidades necessárias para prover uma base segura ao desenvolvimento da gestão e assistência obstétrica. Sendo assim, na formação profissional, destaca-se a importância de disciplinas de organização e gestão nos serviços de saúde no que condiz a conceitualização de princípios básicos e competências gerenciais e administrativas, considerando que o enfermeiro hoje tem assumido de forma estratégica a gerência de equipes e grupos multiprofissionais.

A modalidade residência surge com a proposta da formação pelo trabalho como ensino-aprendizado, e tem como objetivo de formar profissionais de saúde com vistas a superar a segmentação do conhecimento e do cuidado na atenção à saúde. As motivações de sua criação relacionam-se com as políticas de saúde que intentam modificar o modelo obstétrico medicalizado nos serviços, para a instituição de cuidados humanizados, o estímulo ao parto normal e melhores indicadores de qualidade na assistência materna e neonatal (Pereira et al.,2018).

Nessa perspectiva, surge o Programa Nacional de Residência em Enfermagem Obstétrica (PRONAENF), uma ação estratégica que visa a qualificação da assistência às mulheres nos seus processos de saúde reprodutiva, assim como o aumento 
do contingente de profissionais orientados pelas evidências científicas, diretrizes do Sistema Único de Saúde (SUS) e nas recomendações da Rede Cegonha, Política Nacional de Atenção Integral à Saúde da Mulher e Pacto pela redução da Mortalidade.

No estado de Alagoas, a Universidade Estadual de Ciências da Saúde - UNCISAL, em 2005, houve a criação da residência em enfermagem na área de Saúde da Mulher, com carga horária de 40 horas. A partir de 2007, o programa foi expandido, passando a ter 60 horas semanais, sendo as atividades teórico-práticas executadas na instituição hospitalares do complexo UNCISAL e parcerias com Secretaria de Estado da Saúde de Alagoas (SESAU) e Secretaria Municipal de Maceió.

O programa é caracterizado por treinamento em serviço, sob supervisão da Comissão Estadual de Residência em Enfermagem e da PROPEP/UNCISAL possuindo os objetivos: aprimorar habilidades técnicas, o raciocínio clínico e a capacidade de tomar decisões; desenvolver atitude que permita valorizar a significação dos fatores somáticos, psicológicos e sociais que interferem no processo saúde e doença; valorizar as ações de saúde de caráter preventivo; promover a integração do enfermeiro em equipe multiprofissional para prestação de assistência aos pacientes; estimular a capacidade de aprendizagem independente e de participação em programas de educação permanente e estimular a capacidade de crítica de atividade de enfermagem, considerando-a em seus aspectos científicos, éticos e sociais. (UNCISAL, 2019).

Sob as características citadas e intenções do programa, a autora teve a oportunidade de realizar a residência em Enfermagem obstétrica pela Uncisal e posteriormente assumir a liderança de um Centro de Parto Normal em uma Maternidade de baixo risco do Estado de Alagoas.

Diante disso, este estudo teve como objetivo descrever a vivência de uma enfermeira obstétrica egressa pelo programa de residência enquanto líder de um Centro de interligando os conhecimentos e habilidades adquiridas durante a residência com sua implementação na liderança.

\section{Metodologia}

Trata-se de um estudo de natureza qualitativa e descritiva, do tipo relato de experiência de uma egressa pelo Programa de Residência Obstétrica da Uncisal, embasado à vivência de processo de ensino-aprendizagem de competências assistências e gerenciais para enfermagem obstétrica.

A residência ocorreu entre março de 2018 à março de 2020 perpassando por Maternidades de Alto Risco, Risco Habitual e setores de serviço especializado, incluindo os setores como: classificação obstétrica, pré-parto, alojamento conjunto, unidades de internamento de risco, $\mathrm{CCIH}$, núcleo hospitalar de epidemiologia, centro cirúrgico, unidade de terapia intensiva materna, unidade de cuidados intensivos neonatais, assistência pré-natal, serviço de atendimento as doenças sexualmente transmissíveis, além da prática docente em ensino superior acerca do tema saúde da mulher.

Após a conclusão da residência houve o ingresso no mercado de trabalho como enfermeira obstetra assistencial e em julho de 2021 a mesma assumiu a liderança do setor de Centro de Parto em uma Maternidade de Risco Habitual situada no Estado de Alagoas. A instituição citada faz parte do campo de estágio do programa.

Durante a vigência de dois anos no Programa, a autora cumpriu além das atividades práticas uma carga horária de 5.760 horas distruídos entre seminários específicos acerca da Saúde da Mulher e 24 módulos da Residência como um todo, entre disciplinas como: atividades individuais socializadas comunicação e educação em Saúde; gestão em enfermagem, saúde baseada em evidências, políticas públicas em saúde, vigilância em saúde, introdução a libras entre outras. 


\section{Resultados e Discussão}

\subsection{Construindo a base em treinamento}

A inserção no cenário da residência auxiliou a compreender e sanar as lacunas de formação acadêmica. A busca por atualizações e aperfeiçoamento a fim de fortalecer as competências e as habilidades técnicas necessárias para um enfermeiro obstetra assumir a responsabilidade pela organização do serviço perpassou para o gerenciamento inerentes à atividade, bem como o compartilhamento entre os membros da equipe, possibilitando aquisições dos conhecimentos necessários para otimização de uma liderança no serviço.

No decorrer dos dois anos do programa, a vivência nos vários campos contemplados incentivou a tomada de decisões, competência esta estimulada aos residentes a partir do momento que ao atuarem na assistência se deparam com a sua responsabilidade profissional, despindo-se da capa de estagiário para enfermeiro em treinamento.

Esse processo de amadurecimento propicia o gatilho para a importância do planejamento, avaliação de prioridades e realização de procedimentos cabíveis a sua alçada técnica. O qual a primeiro momento se deve a ânsia do saber e aprender uma nova habilidade na prática, mas que se constrói de forma orgânica em um perfil de liderança, necessário para a gestão do cuidado.

Em um estudo realizado com residentes de Alagoas, apontou que desses programas saem profissionais com visão diferenciada e propagadores de uma forma de trabalho (Leão, 2018).

Para alcançarmos a tal ponto, deve-se ser parte da formação acadêmica a utilização de métodos ativos que interligam o saber teórico, construído e aprimorado ao longo de anos, ao cenário prático, valorizando os saberes e vivências apresentadas por cada integrante da equipe. Neste contexto, um dos pontos marcantes para a autora foi a utilização dos módulos do programa da residência abordando a construção e utilização de tecnologias no cuidado da saúde.

Para Merhy (2002), as tecnologias podem ser divididas em: leve — a constituição de relações para implementação do cuidado (vínculo, gestão de serviços e acolhimento) —; leve-dura — a construção do conhecimento por meio de saberes estruturados (teorias, modelos de cuidado, cuidado de enfermagem) - e dura — a utilização de instrumentos, normas e equipamentos tecnológicos.

No decorrer das disciplinas, como: Comunicação em Saúde, Educação em Saúde, Gestão em Enfermagem, Relacionamento interpessoal e interdisciplinaridade, Saúde Baseada em evidências, dentre outras os residentes transpassaram na abordagem aos três tipos de tecnologia.

A relevância deste conhecimento vai de encontro com a Política Nacional de Gestão de Tecnologias em Saúde, estabelecida em 2011 a qual tinha o objetivo de amparar as necessidades dos profissionais para que estes se adaptem à utilização das inovações tecnológicas na área da saúde. Portanto, é de interesse nacional que as tecnologias sejam conhecidas, empregadas e contribuam para a evolução dos serviços prestados nas maternidades, bem como com a redução dos indicadores de saúde materno-infantil (Ministério da Saúde, 2010).

Em um estudo realizado por Salomão et al, (2020) com residentes e preceptores de uma Residência Multiprofissional está inserida em escola vinculada ao SUS do estado do Ceará traz a fala de que os módulos são fundamentais nas intervenções e reflexões sobre a conduta ética em saúde, vivenciada pelos residentes.

Outro ponto empregado no uso das tecnologias nos módulos, foi acerca das tecnologias leves, exemplificando em comunicação entre equipe, profissional/usuário e entre os níveis de atenção à saúde. Isto se depois pois além dos módulos decorrerem de forma interdisciplinar com os demais cursos da saúde contemplados pela Residência Profissional, e não somente a de enfermagem, também envolvia os residentes da saúde coletiva.

A multiprofissionalidade e interdisciplinaridade prediz uma maior eficácia do cuidado, gerada por meio de articulação dos diferentes conhecimentos profissionais (Salomão et al., 2020). 
Para Silva, 2017, a comunicação é o ponto chave para um bom relacionamento dentro dos serviços de saúde. Seja entre a equipe, profissionais e usuários, ou entre os níveis de atenção de saúde, a inclusão dessa tecnologia leve no ambiente da maternidade sinaliza um caminho ideal que tem sido perpassado nessa relação entre o emissor e o receptor. Tal achado indica que as relações têm focado a construção de um pilar para integralidade, equidade e participação de todos nas escolhas destinados a atenção.

A boa comunicação entre profissionais de saúde e os usuários é fundamental, sendo que uma falha nesse processo comumente guia o profissional a uma assistência fragmentada que não supre totalmente as necessidades do usuário (Baquião et al., 2020).

Somatizando o link gerado na associação das aulas teóricas com a assistência, a autora obteve a oportunidade de convites por parte da coordenação de enfermagem do campo prático a oportunidade de desenvolver a educação permanente gerando espaços para ministrar palestras para equipe, além das discussões geradas nos estudos de caso. Porém em todas as situações apenas membros da enfermagem estavam presentes, sem a adesão das demais categorias.

Houveram momentos de barreiras entre o planejamento feito pela residente e a sua implementação. No mais tocante a disparidade ao atendimento a gestante, parturiente e puérpera quanto as intervenções pela equipe médica e de enfermagem. Existia uma falta de comunicação efetiva junto com a categoria, fato potencializado pela ausência de protocolos instituídos na maternidade e ausência de sessões clínicas interdisciplinares.

Esse dilema não trata-se de um problema exclusivo do programa em si ou ao campo prático. Ele é decorrente de anos de um modelo intervencionista e segregador. Podemos visualizar isso em outras literaturas, à exemplo de uma pesquisa qualitativa, descritiva e exploratória, realizada com 13 enfermeiras obstétricas em duas maternidades públicas do Rio de Janeiro. O estudo identificou tensões internas durante a formação, as mesmas revelaram uma questão de espaço territorial, que envolvia a disputa pelo cenário de formação na assistência ao parto normal com residentes da área médica, dificultando a execução do plano sistematizado da assistência em enfermagem prescrito às parturientes. As dificuldades nessa relação entre o conhecimento e o processo de cuidar, assim como as relações profissionais já instituídas, mostraram conflitos no cotidiano da prática das enfermeiras (Silva et al., 2020).

As práticas de formação desenvolvidas nos espaços das residências requerem metodologias que gerem a discussão o olhar crítico acerca do trabalho em saúde nas múltiplas esferas sociais (Alves et al., 2019). Neste processo, destacam-se as rodas de conversa como espaços que viabilizam reflexões acerca das necessidades de saúde, o planejamento e pactuação dos processos de organização de serviço (Lorena et al., 2019).

Outro ambiente que se destacou na experiência da egressa como forte base no desenvolvimento deste olhar crítico e a aplicação da assistência pautada em evidências científicas foi a vivência dos Seminários Acadêmicos ocorridos semanalmente acerca dos assuntos voltados à Saúde da Mulher. Espaço este que propiciou discussões técnica, educação permanente e o incentivo ao tripé educacional (ensino, pesquisa e extensão), além de um momento de compartilhamento de informações e identificações das fragilidades no percurso.

As situações vivenciadas corroboram no processo de formação de uma enfermeira obstetra contribuindo para modificar o paradigma assistencial do modelo obstétrico intervencionista, que possibilita uma transformação qualitativa no cenário obstétrico com resgate sobre a fisiologia, humanização da assistência, integralidade do cuidado, fortalecimento de vínculo, empoderamento da mulher como protagonista, ressignificando o momento do parto e nascimento.

Dentre essas características amadurecidas podemos citar a capacitação a exercer atividades de liderança, para as quais é necessária a autoconfiança respaldada no conhecimento científico para que possa conduzir o atendimento do paciente com segurança. O gerenciamento da equipe, tomada de decisões, solução de conflitos, dentre outros, proveniente da formação de uma líder. 


\subsection{Execução da liderança}

Após a finalização da residência a egressa adentrou no mercado de trabalho assumindo o cargo de enfermeira obstetra de um centro de parto normal no estado de Alagoas, após quatro meses de atuação, a mesma foi convidada a liderança do setor.

No mesmo período o mundo se deparou com o surgimento de uma nova doença, a Coronavirus Disease 2019 (COVID-19), que se tornou um grave problema de saúde pública, repercutindo em diversos desafios para a enfermagem. Em dezembro de 2019, após casos de uma pneumonia atípica registrados na China, na cidade de Wuhan, descobriu-se um novo vírus da família coronavírus, denominado Severe Acute Respiratory Syndrome 2 (SARS-CoV-2), que causa a COVID-19. A partir de então, com a disseminação da doença nos continentes, em 11 de março de 2020, a OMS determinou situação de pandemia mundial (Organização Pan-Americana da Saúde [OPAS], 2020). A Hospital Maternidade tornou-se referência para atendimento ao COVID-19.

Notas de alerta e atualizações eram constantes e a reestruturação do serviço tornou-se rotina. Era essencial por em movimento readequação dos Procedimentos Operacionais Padrão (POP) e criação dos ainda não existentes.

Diante da experiência prévia durante a residência acerca da importância das tecnologias e a interdisciplinaridade houve a criação de parte dos POP's juntamente com a equipe de fisioterapeutas, enfermeiros e higienização. Toda a equipe foi treinada com o apoio da educação permanente da instituição.

Outra característica atípica desde período ocorrida foi o Centro de Parto Normal da devida maternidade obter uma grande queda nas admissões de parturientes devido ao seu perfil ser destinado à gestantes com teste de covid positivo e estarem estáveis. Isso possibilitou um maior espaço para treinamentos e readequações. Oportunizou a criação de dois protocolos (protocolo de admissão - parto adequado e indução ao parto normal) previstos conforme o parecer técnico $\mathrm{n}^{\circ}$ 003/2020 (COFEN,2020).

A possibilidade da construção dos protocolos, bem como a sua implementação permitiu a diminuição de uma fragilidade vista durante a residência e construir ferramentas para uma maior padronização da assistência obstétrica baseadas em evidências científicas, foco de uma gestão otimizada.

O legado de Florence encaminhou o trabalho do profissional enfermeiro para uma atuação pautada no protagonismo técnico-científico, legal e político. Isso só é possibilitado a partir de práticas comprometidas com o bem-estar social nas dimensões de cuidado, gerenciamento e investigação/educação. O fazer cuidativo e gestão da enfermagem necessita de base teórica e evidências científicas (Geremia et al., 2020).

Permeado neste raciocínio, tornou-se importante além dos protocolos e POP's a utilização de um instrumento para a sistematização da assistência de enfermagem (SAE), pois no momento ainda não havia no setor tal instrumento. Sua construção ocorreu a partir da escuta ativa da equipe e embasado nas teorias de enfermagem.

A estratégia de implantação da SAE é um passo importante para o serviço hospitalar sendo potencializado pela adoção institucionalidade uma gestão participativa na qual as pessoas constituem-se sujeitos do processo. Nesse cenário, o centro parto normal como um setor bastante relevante em um hospital maternidade é um ambiente propício para a implantação da SAE (Resende,2017).

Uma pesquisa realizada em uma instituição pública de saúde em Fortaleza, Ceará, Brasil, afirma que quando a SAE é aplicada de forma correta, na totalidade de suas fases, representa um grande benefício, pois além de promover uma assistência mais qualificada, traz também melhorias na organização das atividades de enfermagem e uma maior autonomia profissional, com isso, sua implementação e seu uso deve ser estimulado como uma realidade possível da pratica profissional (Clares, Freitas \& Monnyck, 2012). 
Porém, apesar dos treinamentos para a implementação do instrumento da SAE a falta de compromisso e de conhecimento dos profissionais sobre as etapas da sistematização, registros inadequados da assistência de enfermagem, não adesão as mudanças propostas ocasionaram fragilidades no processo de enfermagem.

Entendeu-se que SAE nas instituições de saúde é algo completamente desafiador, contudo, é preciso esforço da equipe de enfermagem e o suporte da gestão para sistematizar as ações, otimizar o cuidado, evitar ônus desnecessários aos envolvidos, capacitar profissionais da enfermagem e oferecer uma assistência humanizada, baseada na cientificidade das ações, por meio da sistematização das ações assistenciais e gerenciais.

Outra preocupação identificada foi acerca da polarização de ações voltadas apenas ao COVID-19, porém como sabemos as outras patologias e intercorrências seguiam firmes, além da atenção à saúde mental dos colaboradores que seguiam com riscos aumentados ao Burnout devido a sobrecarga e ao isolamento social.

A própria carga horária da residência na época trouxe à margem esta preocupação. A literatura traz relatos de desgaste mental em alguns estudos. Podemos citar uma pesquisa instituída entre profissionais residentes dos Programas de Residência Multiprofissional da Escola de Saúde Pública do Ceará (ESP/CE) em uma mensuração das condições de saúde mental de profissionais residentes do programa. A mesma pode concluir sintomas relacionadas à depressão, raiva, mania, ansiedade, as alterações somáticas, a ideação suicida, a psicose, ao distúrbio do sono, a memória, aos pensamentos, aos comportamentos repetitivos, a dissociação, ao funcionamento da personalidade e ao uso de substância (Pinheiro et al., 2021).

Fato este não somente propícios aos residentes pois conforme estudos a exposição diária dos profissionais de enfermagem frente a situações estressantes, como assistência a pacientes graves, cuidados intensivos e diretos, funções burocráticas e sobrecarga de trabalho, contribui para o desenvolvimento de ansiedade e depressão (Silva et al;2015).

Por isso, é imprescindível que os profissionais de enfermagem se preparem para enfrentar esta ameaça, buscando a informação sobre os fatores de risco e de proteção em relação à pandemia e o que ela acarreta em suas rotinas, tornando-se assim responsabilidade da liderança buscar estratégias de enfrentamento.

Elucidando essa questão, surgiu-se a aplicações de ações permeando reflexões junto com os profissionais e pacientes acerca sentimentos em frente ao combate a pandemia covid e o isolamento social com o suporte dos setores de serviço social e psicologia da instituição de trabalho. No mesmo espaço, oportunizou-se ações educativa junto com alguns membros convidados da equipe médica acerca de cuidados com as demais patologias como prevenção e combate ao câncer de mama e próstata gerando um menor distanciamento entre as classe e diminuição das ações voltadas apenas ao COVID-19.

Em momentos de grandes enfrentamentos, é possível notar que a maioria das pessoas tem força e habilidade para lidar com desafios. Há situações, como a pandemia por Covid-19, que causam estresse e angústia e, para confrontá-las, é possível diagnosticar as estratégias usadas no passado que poderiam ser úteis também para o contexto atual. Embora o cenário seja outro, as estratégias para gestão do estresse podem ser novamente acessadas de modo individual ou coletivo, no intuito de buscar resiliência (OPAS, 2020)

Percebeu-se que momentos como esses reforçam a interligação dos profissionais aos gestores, bem como uma breve análise situacional do emocional e enfrentamento da equipe e pacientes. A importância da escuta ativa é essencial para uma boa liderança aplicada as potencialidades e necessidades do cenário.

\section{Considerações Finais}

Observou-se que a pós graduação na modalidade em Residência em Enfermagem Obstétrica cumpriu seus objetivos, uma vez que possibilitou a aquisição de experiência na assistência obstétrica, permitindo a melhoria do desempenho da egressa na sua assistência como também forneceu o suporte ao exercício da gestão em enfermagem, promovendo a articulação do ensino, pesquisa e assistência.Dessa forma, considera-se a realização de outros estudos que forneçam novos panoramas 
referente a temática abordada, visto que é um campo abrangente e multifatorial, no qual novos conhecimentos poderão fornecer resultados semelhantes ou diferenciados, servindo de base para novas discussões sobre o assunto. Entende-se que a experiências advindas da vivência diária da rotina nos serviços de saúde possibilita o crescimento profissional, olhar crítico, escuta ativa e contextualização do diagnóstico dos riscos reais e potenciais para o embasamento de um planejamento e implementação de ações baseadas na segurança para melhor tomada de decisões frente a situações complexas do trabalho.

\section{Referências}

Aires, R. M. B., \& Guimarães, L. B. E. (2017). Relato de experiência de um grupo de residentes em enfermagem obstétrica sobre práticas de organização do processo de trabalho. Rev enferm UFPE. 11(2). http://repositorio.saude.palmas.to.gov.br/document/view/10

Alves, M. A., Araújo, H. P. A., Costa, L. A. S., Marques, M. C. S., \& Alencar, R. A. (2019). Ação interdisciplinar de promoção à saúde no programa escolar da família: relato de experiência de residentes do programa multidisciplinar em saúde da família. Revista Nursing, 22(252). http://www.revistanursing.com.br/revistas/252/pg31.pdf

Arruda, G. M. S., Barreto, I. C. H. C., Ribeiro, K. G., \& Frota, A. C. (2017). O desenvolvimento da colaboração interprofissional em diferentes contextos de residência multiprofissional em Saúde da Família. Interface. 22(1). https://www.scielo.br/scielo.php?script=sci_arttext\&pid=S1414-32832018000501309

Baquião, L. S. M., Ângelo, S. T., Costa, A. M. B., Dias, A. L. G., \& Constâncio, J. V. B. (2020). Práticas educativas a gestantes em situação de vulnerabilidade social. Brazilian Journal of Development, 22(4). https://www.brazilianjournals.com/index.php/BRJD/article/view/8369/7213

Boaventura, T., Baizer, L. D. C. D., Silva, M. G., Martins, A. R., \& Borges, P. K. O. (2020). Tipologia de Tecnologias em Saúde presentes nas maternidades segundo perspectivas dos profissionais gestores e enfermeiros. Brazilian Journal of Development.6(6). https://www.brazilianjournals.com/index.php/BRJD/article/view/11925/9991

Clares, J. W. B., Freitas M. C., \& Monnyck, H. C. P. (2013). Sistematização da assistência de enfermagem ao idoso institucionalizado fundamentada em Virginia Henderson. Rev Rene. 14(3). http://periodicos.ufc.br/rene/article/view/3505/2746

Geremia, D. S., Vendruscolo, C., Celuppi, I. C., Adamy, Toso, B. R. G. O., \& Souza, J. B. (2020). 200 Anos de Florence e os desafios da gestão das práticas de enfermagem na pandemia COVID-19. Rev. Latino-Am. Enfermagem. 22(3358). https://www.scielo.br/pdf/rlae/v28/pt_0104-1169-rlae-28-e3358.pdf

Leão, S. S. (2018). A interdisciplinaridade na Residência Multiprofissional de uma Universidade Federal do Nordeste: à óptica dos residentes (Dissertação de mestrado). Universidade Federal do Alagoas - UFAL, Maceió AL, Brasil. http://www.repositorio.ufal.br/bitstream/riufal/5882/1/A\%20interdiscipl inaridade $\% 20$ na $\% 20$ resid $\%$ c3\%aancia\%20multiprofissional $\% 20$ de $\% 20$ uma $\% 20$ Universidade $\% 20$ Federal $\% 20$ do $\% 20$ Nordeste $\% 20 \%$ c $3 \%$ a0 $\% 20 \%$ c3\%b3ptica $\% 20$ dos\%20residentes.pdf

Lorena, S. B., Andrade, M. M., Vilela, L. R. A., Arcoverde, A. M. H., \& Sobrinho, J. E. L. (2019). Análise do Acesso à Informação Acadêmica entre Estudantes de Medicina Inseridos numa Metodologia Ativa de Aprendizagem. Revista Brasileira de educação médica. 43(4). https://www.scielo.br/pdf/rbem/v43n4/1981-5271-rbem-43-4-0176.pdf

Merhy, E. E. (2002). Em busca de ferramentas analisadoras das Tecnologias em Saúde: a informação e o dia a dia de um serviço, interrogando e gerindo trabalho em saúde. In: Merhy, E. E., Onoko, R, Agir em saúde: um desafio para o público (2a ed.). 113-50. Hucitec.

Organização Pan-Americana da Saúde. (2020). COVID-19 Intervenções recomendadas em saúde mental e apoio psicossocial (SMAPS) durante a pandemia. Brasil: autor. https://iris.paho.org/bitstream/handle/10665.2/53017/OPASBRANMHMHCOVID-19200026_por.pdf?sequence=1\&isAllowed=y

Oliveira, M. L., \& Silva, N. C. (2012). Estágio de docência na formação do mestre em enfermagem: relato de experiência. Enfermagem em Foco. 3(3). http://revista.cofen.gov.br/index.php/enfermagem/article/view/297

Pereira, A. L. F., Guimarães, J. C. N., Nicácio, M. C., Batista, D. B. S., Mouta, R. J. O., \& Prata, J. A. (2018). Percepções das enfermeiras obstetras sobre sua formação na modalidade de residência e prática profissional. Rev Min Enferm, 22 (1107). http://www.reme.org.br/artigo/detalhes/1243

Pinheiro, C. W., Alves, A. M. S., Carneiro, R. K. M., Muniz, L. G. L., Leal, B. M. V., \& Alencar, A. B. (2021). Panorama de saúde mental de discentes em um programa de residência multiprofissional. J. nurs. health.11(1). https://periodicos.ufpel.edu.br/ojs2/index.php/enfermagem/article/view/19020/12528

Resende, S. L. S. G. Implantação da sistematização da assistência de enfermagem no centro obstétrico do Hospital Materno Infantil Nossa Senhora de Nazareth. (Monografia de Pós-Graduação). Universidade Federal de Minas Gerais -UFMG, Boa Vista RR, Brasil. https://repositorio.ufmg.br/bitstream/1843/33662/1/PI_Sandra.pdf

Silva, D. S. D., Tavares, N. V. S., Alexandre, A. R. G., Freitas, D. A., Brêda, M. Z., \& Melo Neto, V. L. (2015). Depressão e risco de suicídio entre profissionais de Enfermagem: revisão integrativa. Rev. esc. Enferm.49(6). https://www.scielo.br/pdf/reeusp/v49n6/pt_0080-6234-reeusp-49-06-1027.pdf

Silva, G. F., Moura, M. A. V., Martinez, P. A., Souza, I. E. O., Queiroz, A. B. A., \& Pereira, A. L. F. (2020). A formação na modalidade residência em enfermagem obstétrica: uma análise hermenêutico-dialética. Esc. Anna Nery. 24(4). https://www.scielo.br/scielo.php?script=sci_arttext\&pid=S141481452020000400204

Silva, K. S., Santos. A. M., Carvalho, J. A., Kochergin, C. N., \& Almeida, P. F. (2017). Percepção de gestores e enfermeiros sobre a organização do fluxo assistencial na rede de serviços de saúde. - Rev Eletron Comun Inf Inov Saúde. 11(2). https://www.reciis.icict fiocruz.br/index.php/reciis/article/view/1226/2112 
Research, Society and Development, v. 10, n. 11, e459101116499, 2021

(CC BY 4.0) | ISSN 2525-3409 | DOI: http://dx.doi.org/10.33448/rsd-v10i11.16499

Torres, D. B. F. (2020). Programa em Enfermagem Obstétrica: A perspectiva dos residentes. (Dissertação de mestrado). Universidade Federal do Alagoas -

UFAL, Maceió AL, Brasil. http://www.repositorio.ufal.br/bitstream/riufal/7005/3/Programa\%20de\%20resid\%C3\%AAncia\%20de\%20enfermag em\%20 obst\%C3\%A9trica\%3A\% 20a\%20perspectiva\%20dos\%20residentes.pdf 